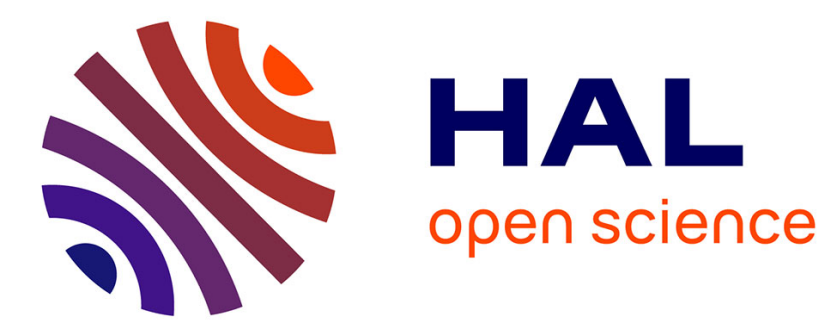

\title{
Collapses and watersheds in pseudomanifolds
}

Jean Cousty, Gilles Bertrand, Michel Couprie, Laurent Najman

\section{To cite this version:}

Jean Cousty, Gilles Bertrand, Michel Couprie, Laurent Najman. Collapses and watersheds in pseudomanifolds. 13th International Workshop on Combinatorial Image Analysis (IWCIA'09), Nov 2009, France. pp.397-410. hal-00622419

\section{HAL Id: hal-00622419 https://hal.science/hal-00622419}

Submitted on 21 Jun 2013

HAL is a multi-disciplinary open access archive for the deposit and dissemination of scientific research documents, whether they are published or not. The documents may come from teaching and research institutions in France or abroad, or from public or private research centers.
L'archive ouverte pluridisciplinaire HAL, est destinée au dépôt et à la diffusion de documents scientifiques de niveau recherche, publiés ou non, émanant des établissements d'enseignement et de recherche français ou étrangers, des laboratoires publics ou privés. 


\title{
Collapses and watersheds in pseudomanifolds
}

\author{
Jean Cousty, Gilles Bertrand, Michel Couprie, and Laurent Najman \\ Université Paris-Est, Laboratoire d'Informatique Gaspard-Monge, A3SI, ESIEE \\ \{j.cousty, g.bertrand, m.couprie, 1.najman\}@esiee.fr
}

\begin{abstract}
This work is settled in the framework of abstract simplicial complexes. We propose a definition of a watershed and of a collapse for maps defined on pseudomanifolds of arbitrary dimension. Through an equivalence theorem, we establish a deep link between these two notions: any watershed can be obtained by collapse iterated until idempotence, and conversely any collapse iterated until idempotence induces a watershed. We also state an equivalence result which links the notions of a watershed and of a collapse with the one of a minimum spanning forest.
\end{abstract}

\section{Introduction}

For topographic purposes, the watershed has been extensively studied during the 19th century [1]. One hundred years later, the watershed transform $[2-5]$ was introduced by Digabel and Lantuéjoul for image segmentation and is now used as a fundamental step in many powerful segmentation procedures [6]. Intuitively, a watershed of a map, seen as a topographic surface, may be thought of as a separating subset of the domain, from which a drop of water can flow down towards several distinct minima.

Topology-preserving transformations, such as homotopic skeletonization $[7$, 8], are used in many applications of image analysis to transform an object while leaving unchanged its topological characteristics. Applications in 2D and 3D are already widely spread. In particular, skeletons are often used as a simplification of the original data, which facilitates shape recognition, registration, or animation. A notion of a homotopic skeleton of a map has also been investigated and was proved to be useful for image processing [9-11]. Intuitively, a map $H$ is said to be homotopic to a map $F$, if every level set of $H$ is homotopic (in the sense of sets) to the corresponding level set of $F$. An (homotopic) ultimate skeleton $H$ of $F$ is a map homotopic to $F$ such that lowering any value of $H$ leads to a map which is no more homotopic to $F$.

Our main result in this paper is an equivalence theorem (Theorem 12) which establishes a deep link between watersheds and homotopy. Intuitively, it states that a set $X$ is a watershed of a map $F$ if and only if there exists an ultimate skeleton $H$ of $F$ such that $X$ is exactly the set of points adjacent to several distinct minima of $H$. This result holds true in a large family of $n$-dimensional discrete spaces, namely the pseudomanifolds. This study is developed in the framework of simplicial complexes (triangulated objects) of arbitrary dimension. 
The notion of watershed that we use here is based on the drop of water principle $[5,12]$ and the one of homotopy relies on the collapse operation [13], a topology-preserving transformation known in algebraic topology. In this context, we furthermore establish (Theorem 13) that any nonempty watershed in a pseudomanifold of dimension $n$ is a simplicial complex of dimension $n-1$. At last, we present a strong link between watersheds, collapses and minimum spanning forests (Theorem 15).

The proofs of the properties presented in this article will be given in an extended version [14]. Notice that all notions and properties can be easily transposed (see [15]) to the framework of cubical complexes, which allows for handling digital images.

\section{Simplicial complexes and pseudomanifolds}

We call (abstract) simplex any finite nonempty set. The dimension of a simplex $x$ is the number of its elements minus one. In the following, a simplex of dimension $d$ will also be called a $d$-simplex. If $x$ is a simplex, we set $\hat{x}=\{y \mid y \subseteq x, y \neq \emptyset\}$. A finite set $X$ of simplices is a cell if there exists $x \in X$ such that $X=\hat{x}$.

Fig. 1a (resp. b,c and d) graphically represents a simplex $x$ of dimension 0 (resp. 1, 2 and 3). Fig. 1e shows a cell composed of one 2-simplex, three 1simplices and three 0 -simplices.

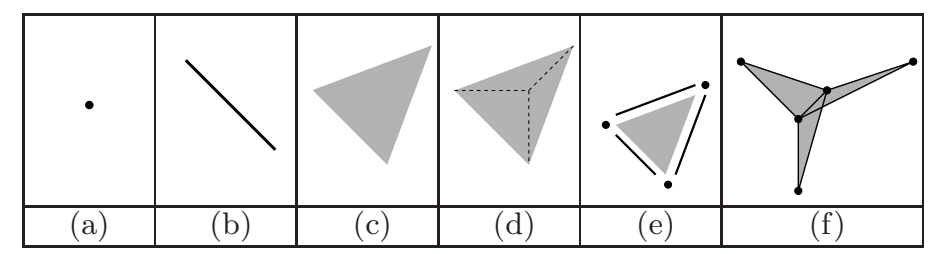

Fig. 1. (a, b, c, d) Simplices of dimension 0, 1, 2 and 3. (e) A 2-cell. (f) A complex.

If $X$ is a finite set of simplices, we write $X^{-}=\bigcup_{x \in X} \hat{x}$, the set $X^{-}$is called the (simplicial) closure of $X$. A finite set $X$ of simplices is a (simplicial) complex if $X=X^{-}$. Let $X$ be a complex. Any element in $X$ is a face of $X$ and we call $d$-face of $X$ any face of $X$ whose dimension is $d$. Any $d$-face of $X$ that is not contained in any $(d+1)$-face of $X$ is called a $(d-)$ facet of $X$. The dimension of $X$ is the largest dimension of its faces. If $d$ is the dimension of $X$, we say that $X$ is pure whenever the dimension of all its facets equals $d$.

Definition 1 A complex $X$ of dimension $n$ is an $n$-pseudomanifold if:

i) $X$ is pure;

ii) any $(n-1)$-face of $X$ belongs to exactly two $n$-faces of $X$;

iii) for any two $n$-faces $x, y$ of $X$, there exists a sequence $\left\langle x_{0}, \ldots, x_{\ell}\right\rangle$ of $n$-faces 
of $X$ such that, $x_{0}=x, x_{\ell}=y$ and, for any $i$ in $\{1, \ldots, \ell\}$, the intersection of $x_{i-1}$ and $x_{i}$ is an $(n-1)$-face of $X$.

The complex of Fig. 1f is pure, its dimension is 2, but it is not a 2-pseudomanifold. Indeed, it contains one 1-face that belongs to three 2 -faces, and six 1-faces that belong to only one 2-face. Fig. 2a shows a subset of a 2-pseudomanifold.

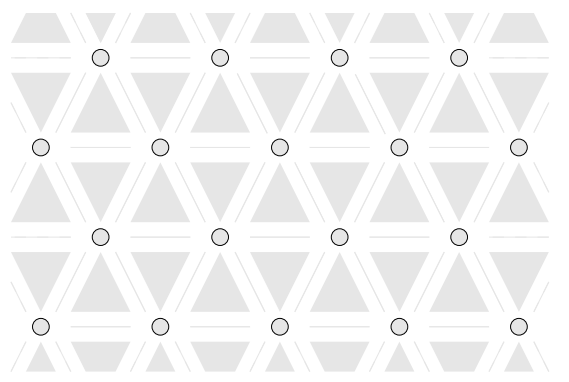

(a)

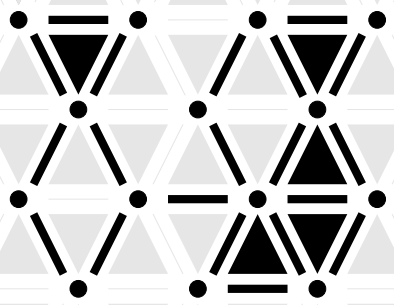

(c)

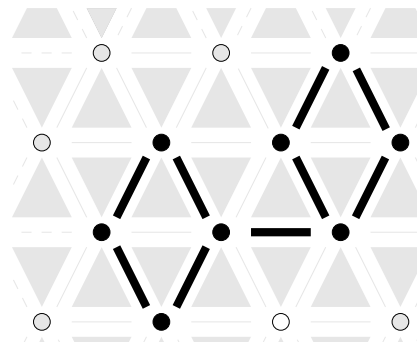

(e)

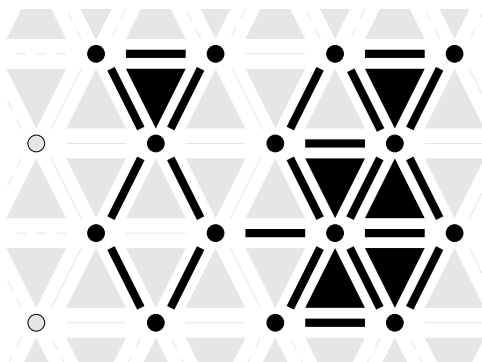

(b)

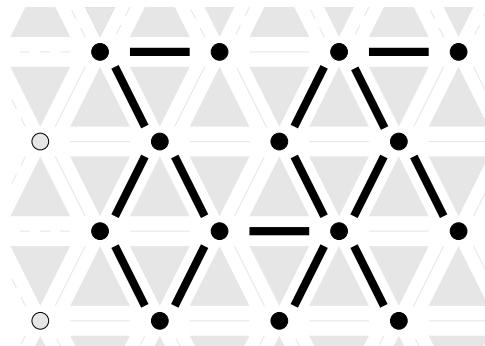

(d)

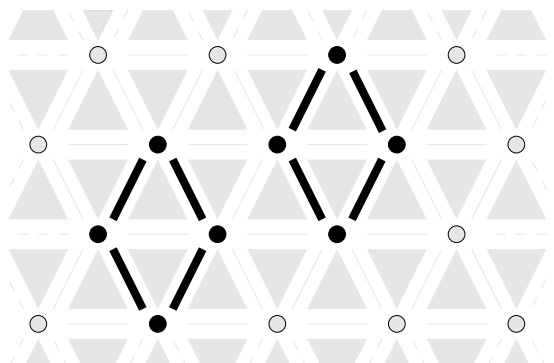

(f)

Fig. 2. (a) A subset of a 2-pseudomanifold $\mathbb{M}$. (b) A subcomplex $X$ of $\mathbb{M}$ in black. (c) An elementary collapse $Y$ of $X$. (d) An ultimate 2-collapse $Z$ of both $X$ and $Y$. (e) An ultimate 1-collapse $W$ of $Z$. (f) A cut for $\bar{X}$. 
Important notation. In this paper $\mathbb{M}$ stands for any $n$-pseudomanifold, where $n$ is a positive integer.

Any subset of a complex $X$ which is also a complex is called subcomplex of $X$. If $Y$ is a subcomplex of $X$, we write $Y \preceq X$. If $X \subseteq \mathbb{M}$, we denote by $\bar{X}$ the complementary set of $X$ in $\mathbb{M}$, i.e. $\bar{X}=\mathbb{M} \backslash X$. Note that if $X \preceq \mathbb{M}, \bar{X}$ is, in general, not a complex.

\section{Collapse}

In this section, we present the operation of collapse introduced by J.H.C. Whitehead [13], which is a discrete analogue of a retraction, that is, a continuous (homotopic) deformation of an object onto itself. This operation defined on the simplicial complexes is similar to the one of removal of a simple point defined on the subsets of the grid points $\mathbb{Z}^{2}$ and $\mathbb{Z}^{3}$ in the context of digital topology [7].

Let $X \preceq \mathbb{M}, y$ be any face of $X$ and $d-1$ be the dimension of $y$. If there exists a unique face $x$ of $X$ which strictly contains $y$, we say that $y$ is a free face for $X$ and that the pair $(x, y)$ is a free pair or a free $d$-pair for $X$.

Let $X \preceq \mathbb{M}$. If $(x, y)$ is a free pair for $X$, the complex $X \backslash\{x, y\}$ is an elementary collapse of $X$ or, more precisely, an elementary $d$-collapse if $(x, y)$ is a free $d$-pair.

Let $X$ and $Y$ be two subcomplexes of $\mathbb{M}$. The complex $Y$ is a collapse of $X$ if there exists a collapse sequence from $X$ to $Y$, i.e., a sequence of complexes $\left\langle X_{0}, \ldots, X_{\ell}\right\rangle$ such that $X_{0}=X, X_{\ell}=Y$ and $X_{i}$ is an elementary collapse of $X_{i-1}$, for any $i$ in $\{1, \ldots, \ell\}$. If each $X_{i}$ is an elementary $d$-collapse of $X_{i-1}$, we also say that $Y$ is a $d$-collapse of $X$. If $Y$ is a $d$-collapse of $X$ that does not contain any free $d$-pair for $Y, Y$ is called an ultimate $d$-collapse of $X$.

Let $X, Y, Z$ and $W$ be the four subcomplexes in black in Figs. 2b,c,d and e respectively. The complex $Y$ is an elementary 2-collapse of $X$. The complex $Z$ is an ultimate 2-collapse of both $X$ and $Y$, and $W$ is a collapse of $X, Y$ and $Z$ which is also an ultimate 1-collapse of $Z$.

\section{Cuts}

Segmentation is the task of delineating objects of interest. In many cases, the result of such a process is a set of connected regions lying in a background which constitutes the separation between regions. Intuitively, a separation which cannot be reduced without connecting some regions is a cut. Our aim being to study segmentations in pseudomanifolds, we introduce hereafter the notions of connected components and cuts of a set of simplices. Then, we present two important results. First, we state (Property 3 ) that in an $n$-pseudomanifold the dimension of a nonempty cut is always $n-1$. Secondly, we give an equivalence result (Theorem 5) between cuts and some subsets of the ultimate collapses. This last property leads to an efficient method to compute cuts in pseudomanifolds.

Let $A \subseteq \mathbb{M}$. Let $\pi=\left\langle x_{0}, \ldots, x_{\ell}\right\rangle$ be an ordered sequence of simplices in $A, \pi$ is a path from $x_{0}$ to $x_{\ell}$ in $A$ if, for any $i \in\{1, \ldots, \ell\}$, either $x_{i-1} \subseteq x_{i}$ or $x_{i} \subseteq x_{i-1}$. 
The set $A$ is said to be connected if, for any two simplices $x$ and $y$ of $A$, there exists a path from $x$ to $y$ in $A$. Let $B \subseteq A$. We say that $B$ is a connected component of $A$ if $B$ is a connected subset of $A$ which is maximal for this property, i.e., for any connected subset $C$ of $\mathbb{M}, B \subseteq C \subseteq A$ implies $C=B$.

Definition 2 Let $A$ and $B$ be two nonempty sets of simplices in $\mathbb{M}$.

We say that $B$ is an extension of $A$ if $A \subseteq B$, and if each connected component of $B$ contains exactly one connected component of $A$. We also say that $B$ is an extension of $A$ if $A$ and $B$ are both empty.

Let $X \subseteq \mathbb{M}$. We say that $X$ is a cut for $A$ if $\bar{X}$ is an extension of $A$ and if $X$ is minimal for this property, i.e., for any $Y \subseteq X, \bar{Y}$ is an extension of $A$ implies $Y=X$.

For instance, the sets of gray simplices in Figs. 2c-f are extensions of the set $A$ of gray simplices in Fig. 2b. The set of black simplices in Fig. $2 \mathrm{f}$ is a cut for $A$.

Intuitively, a "frontier" or a cut in an $n$-dimensional space should be an object of dimension $n-1$. Nevertheless, we have shown [16] that the cuts (also called cleft in [16]) in the grids $\mathbb{Z}^{2}, \mathbb{Z}^{3}, \mathbb{Z}^{n}$ equipped with usual adjacency relations [7] cannot be considered as $(n-1)$-dimensional objects (the cuts are not necessarily thin). From this point of view, the next result shows that the framework of pseudomanifolds is an interesting alternative to usual adjacency relations.

Property 3 Let $A$ and $X$ be two subsets of $\mathbb{M}$ such that $\bar{A}$ and $X$ are two complexes. If $X$ is a cut for $A$, then the complex $X$ is either empty, or is a pure $(n-1)$-complex. Furthermore, $X$ does not contain any free $n$-pair.

Let $X \subseteq \mathbb{M}$. We say that a face $x$ of $\mathbb{M}$ is adjacent to $X$ if there exists a simplex $y$ in $X$ such that either $x \subseteq y$ or $y \subseteq x$. A simplex $x$ in $X$ which is adjacent to more than one connected component of $\bar{X}$ is said to be multiconnected for $X$.

Definition 4 Let $A \subseteq \mathbb{M}$ such that $\bar{A}$ is a complex. Let $X$ be an ultimate $n$ collapse of $\bar{A}$ and let $Y$ be the complex made of all faces which are multi-connected for $X$. We say that the complex $Y$ is a cut by collapse for $A$.

Following Section 3 , in order to compute an ultimate $n$-collapse, we have to iterate steps of elementary collapse, until stability. Each step of elementary collapse requires only a local test. Moreover, the use of a breadth first strategy leads to a linear-time algorithm for computing ultimate collapses. However, the notion of a cut relies on a criterion, which is linked to connected components of the complement, and that requires a global computation (connected components labeling). Remark that the computation of a cut by collapse requires only one step of global computation: a labeling which can also be performed in lineartime. The following result establishes that any complex which is a cut, is a cut by collapse and that the converse is also true. Therefore, the above strategy constitutes a simple and linear-time algorithm to compute cuts.

Theorem 5 Let $A \subseteq \mathbb{M}$ such that $\bar{A}$ is a complex. A complex $X$ is a cut for $A$ if and only if it is a cut by collapse for $A$. 
Remark 6 Let $A \subseteq \mathbb{M}$. Note that if $\bar{A}$ is a complex, then there always exist a cut for A which is a complex. Indeed, in this case there exists by construction a complex which is a cut by collapse for $A$ and which is also, by Theorem 5, a cut for A. For instance, the complex in black in Fig. 2f is both a cut and a cut by collapse for A (in gray in Fig. 2b). On the other hand, if $\bar{A}$ is not a complex, there does not necessarily exist a cut for $A$ which is a complex. For instance, the only cut (in black, Fig. 3b) for the set of gray simplices in Fig. 3 a is not a complex. Observe also that this object contains a face of dimension 2, which would not be the case if $\bar{A}$ were a complex (see Property 3).

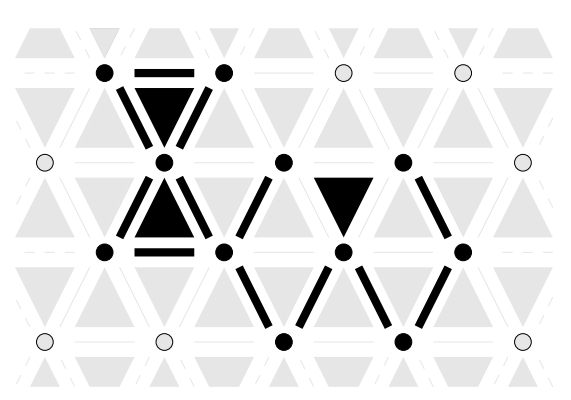

(a)

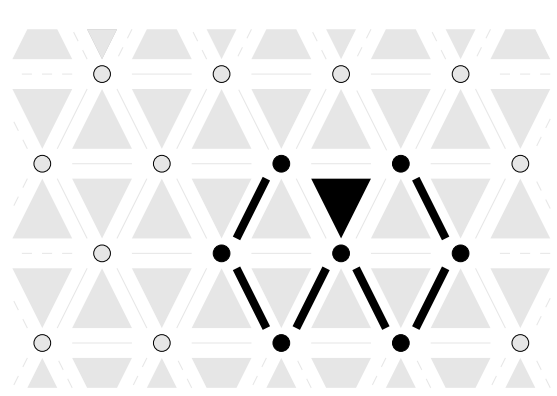

(b)

Fig. 3. Illustration of Remark 6. (a) A set $A$ of simplices such that $\bar{A}$ (in black) is not a complex. (b) In black, the only cut for $A$.

\section{$5 \quad$ Simplicial stacks}

This section presents some basic definitions relative to maps defined on a pseudomanifold. In particular, we introduce the simplicial stacks as the maps whose level sets are all simplicial complexes. This notion will be used in the next section to easily extend the operation of collapse from complexes to maps.

Here and subsequently $k_{\min }$ and $k_{\max }$ stand for two elements of $\mathbb{Z}$ such that $k_{\min }<k_{\max }$. We set $\mathbb{K}=\left\{k \in \mathbb{Z} \mid k_{\min } \leq k \leq k_{\max }\right\}$.

Let $F$ be any map from $\mathbb{M}$ into $\mathbb{K}$. For any face $x$ of $\mathbb{M}$, the value $F(x)$ is called the altitude of $x$ (for $F$ ). Let $k \in \mathbb{K}$. The $k$-section of $F$, denoted by $F[k]$, is the set of faces of $\mathbb{M}$ whose altitude is greater than or equal to $k: F[k]=\{x \in \mathbb{M} \mid F(x) \geq k\}$.

Let $F$ be any map from $\mathbb{M}$ into $\mathbb{K}$, let $A \subseteq \mathbb{M}$ and let $k \in \mathbb{K}$. We say that $A$ is a minimum of $F$ (at altitude $k$ ) if $A$ is a connected component of $\overline{F[k+1]}$ and if $A \cap \overline{F[k]}=\emptyset$. In the following, we denote by $M(F)$, the union of all minima of $F$ and by $\bar{M}(F)$ its complementary set.

A desirable property is that any watershed of a stack $F$ be both a complex and a cut for $M(F)$. However, as noted in Remark 6 , if $\bar{M}(F)$ is not a complex, 
we cannot ensure that such a watershed exists. The notion of a simplicial stack, introduced hereafter, ensures that $\bar{M}(F)$ is a complex and thus that there exists a watershed of $F$ which satisfies the above property.

Definition $7 A$ (simplicial) stack $F$ (on $\mathbb{M}$ ) is a map from $\mathbb{M}$ into $\mathbb{K}$ such that, for any $k \in \mathbb{K}$, the $k$-section of $F$ is a simplicial complex.

Fig. 5a depicts a stack $F$ and Figs. 5b, c and d depict in black the $k$-sections of $F$ for respectively $k=1,2,3$.

Remark 8 Observe that we can obtain a stack $H$ from any map $F$ by considering the simplicial closure of $F$, i.e., $H$ is the simplicial stack such that, for any $k \in \mathbb{K}$, the $k$-section of $H$ is the simplicial closure of the $k$-section of $F$. Note that the simplicial closure $H$ of a map $F$ can be easily obtained by setting $H(x)$ to the maximum altitude for $F$ of the simplices which contain $x$ (i.e. $H(x)=\max \{F(y) \mid x \subseteq y$ and $y \in \mathbb{M}\}$ ), for any face $x$ of $\mathbb{M}$.

Important notation. In the sequel, $F$ denotes a simplicial stack on $\mathbb{M}$.

\section{Collapse of simplicial stacks}

We propose an operation of a collapse of a simplicial stack based on the collapse operation in the sections of the stack. In this framework, an ultimate collapse of a stack can be seen as an analog of a homotopic grayscale skeleton in digital topology $[9,11]$.

Let $y$ be any face of $\mathbb{M}, d-1$ be the dimension of $y$ and $k=F(y)$. If $y$ is a free face for $F[k]$, we say that $y$ is a free face for $F$. If $y$ is a free face for $F$, there exists a unique face $x$ in $F[k]$ such that $(x, y)$ is a free pair for $F[k]$ and we say that the pair $(x, y)$ is a free pair or a free $d$-pair for $F$.

In Fig. 5a, the 1-face $y$ at altitude 1 is a free face for the depicted map $F$. Indeed, $y$ is a free face for $F[1]$ (Fig. 5b). Thus, the pair $(x, y)$ in Fig. 5a is a free pair for $F$. Note that, for any stack $F$, if $(x, y)$ is a free pair for $F$, then $F(x)=F(y)$.

If $(x, y)$ is a free pair for $F$, the simplicial stack $H$ defined by $H(x)=H(y)=$ $F(x)-1$ and by $H(z)=F(z)$ for any $z \in \mathbb{M} \backslash\{x, y\}$ is called an elementary collapse of $F$ or, more precisely, an elementary $d$-collapse of $F$ if $(x, y)$ is a free $d$-pair.

Let $H$ be a simplicial stack on $\mathbb{M}$. We say $H$ is a collapse of $F$ if there exists a collapse sequence from $F$ to $H$, i.e., a sequence of stacks $\left\langle F_{0}, \ldots, F_{\ell}\right\rangle$ such that $F_{0}=F, F_{\ell}=H$ and $F_{i}$ is an elementary collapse of $F_{i-1}, i \in\{1, \ldots, \ell\}$. If each $F_{i}$ is an elementary $d$-collapse of $F_{i-1}$, we also say that $H$ is a $d$-collapse of $F$. If $H$ is a $d$-collapse of $F$ which does not contain any free $d$-pair, $H$ is called an ultimate $d$-collapse of $F$.

The stack $H$ (Fig. 5e) depicts an ultimate 2-collapse of the map F (Fig. 5a).

Remark 9 Note that a stack $H$ is a collapse of $F$ if and only if, for any $k \in \mathbb{K}$, the $k$-section of $H$ is a collapse of the $k$-section of $F$. In this sense, we can say 
that the operation of collapse in simplicial stacks extends the one on simplicial complexes. In particular, if $F$ is an indicator map of a complex $X$ (i.e. $a$ simplicial stack such that the altitude of the faces of $X$ equals 1 and the altitude of the faces in $\bar{X}$ equals 0 ), it is equivalent to consider a collapse of $F$ or to consider the indicator map of a collapse of the complex $X$.

\section{Watersheds}

We now introduce the notion of a watershed in a pseudomanifold. Then, we present the main result (Theorem 12) of the section which establishes an equivalence between the watersheds of a map $F$ and some sets of faces obtained from the ultimate collapses of $F$. In consequence, we can straightforwardly extract a watershed of a stack $F$ from any ultimate collapse of $F$ and, conversely, any watershed of $F$ can be extracted from an ultimate collapse of $F$.

Intuitively, the "catchment basins" of a watershed constitute an extension of the minima and they are separated by a cut from which a drop of water can flow down towards distinct minima. Thus, before defining watersheds, we need the notion of a descending path.

Let $\pi=\left\langle x_{0}, \ldots, x_{\ell}\right\rangle$ be a path in $\mathbb{M}$. We say that the path $\pi$ is descending (for $F$ ) if, for any $i \in\{1, \ldots, \ell\}, F\left(x_{i}\right) \leq F\left(x_{i-1}\right)$.

Definition 10 Let $X \preceq \mathbb{M}$ be a cut for $M(F)$. We say that $X$ is a watershed of $F$ if, for any $x \in \bar{X}$, there exist two descending paths $\pi_{1}=\left\langle x, x_{0}, \ldots, x_{\ell}\right\rangle$ and $\pi_{2}=\left\langle x, y_{0}, \ldots, y_{m}\right\rangle$ such that:

- $x_{\ell}$ and $y_{m}$ are simplices of two distinct minima of $F$; and

- $x_{i} \notin X, y_{j} \notin X$, for any $i$ in $\{0, \ldots, \ell\}$ and $j \in\{0, \ldots, m\}$.

For instance, the set of black faces in Fig. $5 \mathrm{f}$ is a watershed of the map $F$ (Fig. 5a).

We call divide of a stack $F$ the set $\bar{M}(F)$ of all faces of $\mathbb{M}$ which do not belong to any minimum of $F$. Observe that the divide of an ultimate collapse $H$ of $F$ is located on the "crests" of $F$ (see, for instance, the non-null faces in Fig. 5e). On the other hand, we can say intuitively that a watershed of $F$ corresponds to the "closed contours" located on the "crests" of $F$. Hence, a desirable property is that the watersheds of $F$ correspond to the "closed contours" of the divide of the ultimate collapses of $F$. The following theorem asserts that this intuitive property is indeed true in the present framework.

Definition 11 Let $X \subseteq \mathbb{M}$. We say that $X$ is a cut by collapse for $F$ if there exist an ultimate $n$-collapse $H$ of $F$ such that $X$ is the set of all multi-connected elements for $\bar{M}(H)$.

The next theorem, which is one of our main results, generalizes Theorem 5 to the case of stacks. It establishes a deep link between watersheds and homotopy.

Theorem 12 Let $X \subseteq \mathbb{M}$. The set $X$ is a watershed of $F$ if and only if $X$ is a cut by collapse for $F$. 
As far as we know, a similar property is not verified in other discrete frameworks. In particular, in digital topology, there is no such a straightforward relation between topological watersheds [4] and grayscale homotopic skeletons [9]. This point will be developed in a forthcoming extended version of the paper. On the other hand, a similar statement, linking watersheds and skeletons by influence zones, was presented by Najman and Schmitt [17] in the framework of continuous $\left(\mathcal{C}^{2}\right)$ maps.

Remark that, there always exists a watershed of $F$. Indeed, since $F$ is a stack, there exists by construction a cut by collapse for $F$ which, by Theorem 12 , is also a watershed of $F$. Furthermore, any watershed of $F$ is by definition a complex and a cut for $M(F)$. Thus, we deduce the following result from Property 3 .

Theorem 13 Any watershed of $F$ is either empty or is a pure $(n-1)$-complex.

\section{Minimum spanning forests}

In this section, we establish a link between the framework studied in this paper and the one of watersheds and minimum spanning forests in edge-weighted graphs $[5,12]$. To this end, we first recall the definition of a minimum spanning forest relative to a graph. We will see that each of these forests induces a graph cut. Then, we propose a simple construction to derive a graph from $\mathbb{M}$ and we show the equivalence between the watersheds in $\mathbb{M}$ and the graph cuts induced by the minimum spanning forests relative to the minima in this graph. An important consequence is that the efficient algorithms to compute watersheds $[5,12]$ and minimum spanning forests in edge-weighted graphs can be used to compute watersheds and collapses in simplicial complexes.

A graph is a simplicial complex of dimension 1 . If $G$ is a graph, we often consider the pair $(V(G), E(G))$ where $V(G)$ and $E(G)$ are respectively the set of 0 -faces of $G$ and the set of 1-faces of $G$. If $G$ is a graph, any element of $V(G)$ is called a vertex or a point of $G$ and any element of $E(G)$ is called an edge of $G$.

Let $A \subseteq \mathbb{M}$. We define the graph $G_{A}$ such that the vertex set of $G_{A}$ is composed of all $n$-simplices of $A$ and such that two $n$-simplices $x, y$ of $A$ form an edge $\{x, y\}$ of $G_{A}$ if $x \cap y$ is an $(n-1)$-simplex which belongs to $A$.

Important notation. In the sequel, we consider the graph $G_{\mathbb{M}}$ and the map $F_{G}$, from $E\left(G_{\mathbb{M}}\right)$ into $\mathbb{K}$, defined by $F_{G}(\{x, y\})=F(z)$ where $z=x \cap y$, for any $\{x, y\} \in E\left(G_{\mathbb{M}}\right)$. The pair $\left(G_{\mathbb{M}}, F_{G}\right)$ forms an edge-weighted graph.

For instance, the edge-weighted graph $\left(G_{\mathbb{M}}, F_{G}\right)$ associated with the pseudomanifold $\mathbb{M}$ and the stack $F$ presented in Fig. 5a is shown in Fig. $5 \mathrm{~g}$.

Let us now introduce the notion of a spanning forest relative to a subgraph of $G_{\mathbb{M}}$. Generally, in graph theory, a forest is defined as a graph that does not contain any cycle. In this paper, the notion of forest is not sufficient since we want to deal with extensions of subgraphs that can contain cycles (such as the graph obtained from the minima of $F$ ). Therefore, we present hereafter the notion of a relative forest. Intuitively, a forest relative to a subgraph $A$ of $G_{\mathbb{M}}$ is an extension $B$ of $A$ such that any cycle in $B$ is also a cycle in $A$. In other 
words, to construct a forest relative to an arbitrary subgraph $A$ of $G_{\mathbb{M}}$, one can add edges to $A$, provided that the added edges do not introduce new cycles and that the obtained graph remains an extension of $A$. Formally, the notion of cycle is not necessary to define a relative forest.

Let $A$ and $B$ be two nonempty subgraphs of $G_{\mathbb{M}}$. We say that $B$ is a forest relative to $A$ if:

i) $B$ is an extension of $A$; and

ii) for any extension $C \subseteq B$ of $A$, we have $C=B$ whenever $V(C)=V(B)$.

We say that $B$ is a spanning forest relative to $A$ (for $G_{\mathbb{M}}$ ) if $B$ is a forest relative to $A$ and if $V(B)=V\left(G_{\mathbb{M}}\right)$.

Informally speaking, condition ii) imposes that we cannot remove any edge from $B$ while keeping an extension of $A$ that has the same vertex set as $B$.

Remark that if the set $A$ in the previous definition is a set of isolated vertices, we retrieve the usual notion of a forest.

Let $A$ be a subgraph of $G_{\mathbb{M}}$, the weight of $A$ (for $F_{G}$ ), denoted by $F_{G}(A)$, is the sum of the weights of the edges in $E(A): F_{G}(A)=\sum_{u \in E(A)} F_{G}(u)$.

Definition 14 Let $A$ and $B$ be two subgraphs of $G_{\mathbb{M}}$. We say that $B$ is a minimum spanning forest (MSF) relative to $A$ (for $F_{G}$, in $G_{\mathbb{M}}$ ) if $B$ is a spanning forest relative to $A$ and if the weight of $B$ is less than or equal to the weight of any other spanning forest relative to $A$.

For instance, the graph made of bold edges and vertices in Fig. 5h is an MSF relative to the bold subgraph $B$ of Fig. 5g.

The following theorem states that the graph associated with the minima of an ultimate $n$-collapse of $F$ is an MSF relative to the graph associated with the minima of $F$. More remarkably, any MSF relative to the graph associated with the minima of $F$ can be obtained thanks to an ultimate $n$-collapse of $F$. In this sense, the ultimate $n$-collapses on stacks can be said optimal.

Theorem 15 Let $A$ be a subgraph of $G_{\mathbb{M}}$. The graph $A$ is an MSF relative to the graph $G_{M(F)}$ if and only if there exists an ultimate $n$-collapse $H$ of $F$ such that $A=G_{M(H)}$.

As a corollary of Theorem 15, we now establish the link between the watersheds in $\mathbb{M}$ and the minimum spanning forests in $G_{\mathbb{M}}$.

Let $A$ be a subgraph of $G_{\mathbb{M}}$ and let $X$ be a set of edges of $G_{\mathbb{M}}$. We say that $X$ is an MSF cut for $A$, if there exists an MSF $B$ relative to $A$ such that $X$ is the set of all edges of $G_{\mathbb{M}}$ adjacent to two distinct connected components of $B$. $X\}$.

If $X$ is a set of $(n-1)$-faces of $\mathbb{M}$, we set $E d g e(X)=\left\{\{x, y\} \in E\left(G_{\mathbb{M}}\right) \mid x \cap y \in\right.$

Theorem 16 Let $X$ be a set of $(n-1)$-faces of $\mathbb{M}$. The complex $X^{-}$is a watershed of $F$ if and only if $E d g e(X)$ is an MSF cut for $G_{M(F)}$.

This theorem is illustrated in Fig. 5. Let $Y$ be the set of 1-faces depicted in black in Fig. 5f. The complex $X=Y^{-}$(Fig. 5f) is a watershed of the stack $F$ 
(Fig. 5a) and the set $\operatorname{Edge}(Y)$ (dashed edges in Fig. 5h) is an MSF cut for the graph $G_{M(F)}$ (shown in bold in Fig. 5g).

In consequence of Theorem 16, it should be noted that, to compute a watershed of a stack $F$, it is sufficient to compute, in the graph $G_{\mathbb{M}}$, an MSF cut relative to the graph associated with the minima of $F$. An MSF cut can be computed using any minimum spanning tree algorithm (see [5]). The best complexity for solving this problem is reached by the quasi linear-time algorithm of Chazelle [18]. In fact, the problem of computing MSFs relative to the minima is simpler. In [5,12], two linear-time algorithms to compute MSF cuts relative to the minima are proposed. These two algorithms do not require any sorting step, or the use of a hierarchical queue or a representation to maintain unions of disjoint sets. Thanks to Theorems 15 and 16, these algorithms can be used to compute, in linear-time, ultimate $n$-collapses and watersheds in pseudomanifolds. The position of the contours produced by watershed algorithms on the plateaus is the subject of many discussions (see e.g. $[17,19,20]$ ). Depending on the implementation, the algorithm proposed in [12] allows for placing the watersheds either on the border of the plateaus or in the "middle". It is also possible to define explicitely the locus of the watershed on plateaus. For example, the algorithm presented in [21] allows for an optimal placement of the watershed on the plateaus with respect to random walkers criterion [22] or min cut criterion [23].

\section{Conclusion}

In this paper, we introduce the simplicial stacks as the maps on pseudomanifolds whose level sets are all simplicial complexes. Then, we propose definitions of watersheds and collapses for simplicial stacks. We establish a deep link between these two notions and also with the notion of a relative minimum spanning forest. The proposed framework can be applied for segmenting the triangulated surface of 3D objects (see, e.g., [24] and Fig. 4). It can also be easily transposed to cubical complexes which allows for handling digital images [15].

\section{References}

1. Maxwell, J.: On hills and dales. Philosophical Magazine 4/40 (1870) 421-427

2. Digabel, H., Lantuéjoul, C.: Iterative algorithms. In: 2nd European Symp. Quantitative Analysis of Microstructures in Material Science, Biology and Medicine. (1978) 85-89

3. Vincent, L., Soille, P.: Watersheds in digital spaces: An efficient algorithm based on immersion simulations. IEEE Transactions on Pattern Analysis and Machine Intelligence 13(6) (1991) 583-598

4. Bertrand, G.: On topological watersheds. Journal of Mathematical Imaging and Vision 22(2-3) (2005) 217-230

5. Cousty, J., Bertrand, G., Najman, L., Couprie, M.: Watershed cuts: Minimum spanning forests and the drop of water principle. IEEE Transactions on Pattern Analysis and Machine Intelligence 31(8) (2009) 1362-1374 


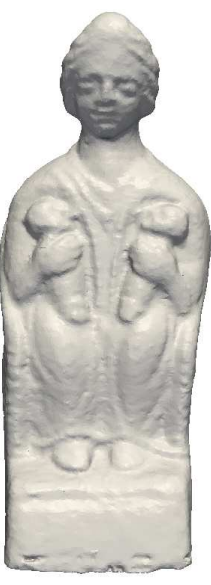

(a)

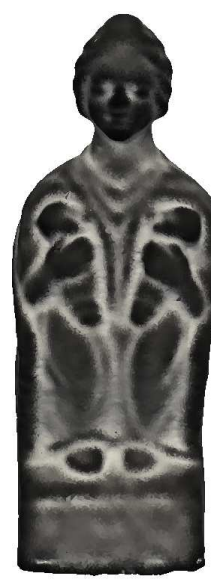

(b)

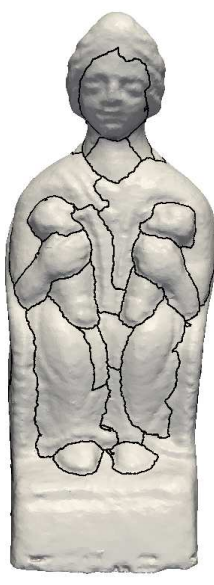

(c)

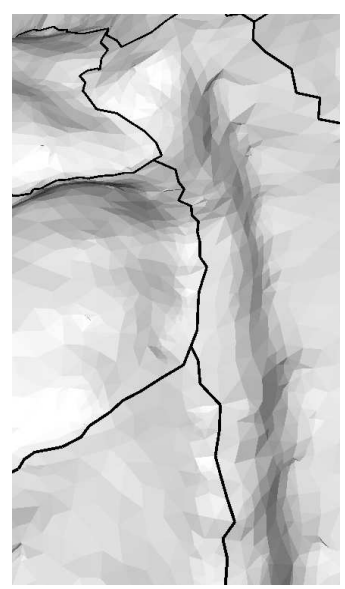

(d)

Fig. 4. (a) Rendering of a 2-pseudomanifold $\mathbb{M}$. (b) a simplicial stack $F$ on $\mathbb{M}$ (which behaves like the inverse of the mean curvature of the surface, see [24]) (c) A watershed (in black) of $F$. (d) Zoom on a part of (c). The object $\mathbb{M}$ shown in (a) is provided by the French Museum Center for Research)

6. Beucher, S., Meyer, F.: The morphological approach to segmentation: the watershed transformation. E. Dougherty (Ed.), Mathematical Morphology in Image Processing, Marcel Decker (1993) 443-481

7. Kong, T., Rosenfeld, A.: Digital topology: Introduction and survey. Computer Vision, Graphics, and Image Processing 48(3) (1989) 357-393

8. Couprie, M., Bertrand, G.: New characterizations of simple points in 2D, 3D, and 4D discrete spaces. IEEE Transactions on Pattern Analysis and Machine Intelligence 31(4) (2009) 637-648

9. Bertrand, G., Everat, J.C., Couprie, M.: Image segmentation through operators based upon topology. Journal of Electronic Imaging 6(4) (1997) 395-405

10. Couprie, M., Bezerra, F.N., Bertrand, G.: Topological operators for grayscale image processing. Journal of Electronic Imaging 10(4) (2001) 1003-1015

11. Ranwez, V., Soille, P.: Order independent homotopic thinning for binary and grey tone anchored skeletons. Pattern Recognition Letters 23(6) (2002) 687 - 702

12. Cousty, J., Bertrand, G., Najman, L., Couprie, M.: Watershed cuts: thinnings, shortest-path forests and topological watersheds. IEEE Transactions on Pattern Analysis and Machine Intelligence (2009) to appear.

13. Whitehead, J.H.C.: Simplicial Spaces, Nuclei and m-Groups. Proc. London Math. Soc. s2-45(1) (1939) 243-327

14. Cousty, J., Bertrand, G., Couprie, M., L., N.: Watersheds and collapses in pseudomanifolds of arbitrary dimension. In preparation.

15. Bertrand, G., Couprie, M., Cousty, J., Najman, L.: Chapitre 4 : Ligne de partage des eaux dans les espaces discrets. In: Morphologie mathématique : approches déterministes. Hermes Sciences Publications (2008) 123-149 
16. Cousty, J., Bertrand, G., Couprie, M., Najman, L.: Fusion graphs: merging properties and watersheds. Journal of Mathematical Imaging and Vision 30(1) (2008) 87-104

17. Najman, L., Schmitt, M.: Watershed of a continuous function. Signal Processing 38(1) (1993) 68-86

18. Chazelle, B.: A minimum spanning tree algorithm with inverse-Ackermann type complexity. Journal of the ACM 47 (2000) 1028-1047

19. Roerdink, J.B.T.M., Meijster, A.: The watershed transform: Definitions, algorithms and parallelization strategies. Fundamenta Informaticae 41(1-2) (2001) $187-228$

20. Audigier, R., Lotufo, R.: Uniquely-determined thinning of the tie-zone watershed based on label frequency. Journal of Mathematical Imaging and Vision 27(2) (2007) 157-173

21. Couprie, C., Grady, L., Najman, L., Talbot, H.: Power watersheds: A new image segmentation framework extending graph cuts, random walker and optimal spanning forest. In: International Conference on Computer Vision, IEEE (2009) To appear.

22. Grady, L.: Random walks for image segmentation. IEEE Transactions on Pattern Analysis and Machine Intelligence 28(11) (2006) 1768-1783

23. Boykov, Y., Veksler, O., Zabih, R.: Fast approximate energy minimization via graph cuts. IEEE Transactions on Pattern Analysis and Machine Intelligence 23(11) (2001) 1222-1239

24. Alcoverro, M., Philipp-Foliguet, S., Jordan, M., Najman, L., Cousty, J.: Regionbased artwork indexing and classification. In: Proc. 2-nd 3DTV-conference, IEEE (2008) 393-396 


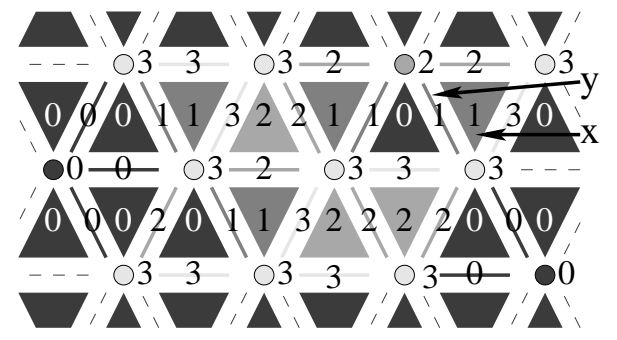

(a)

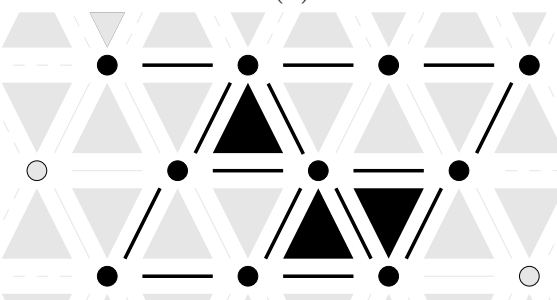

(c)

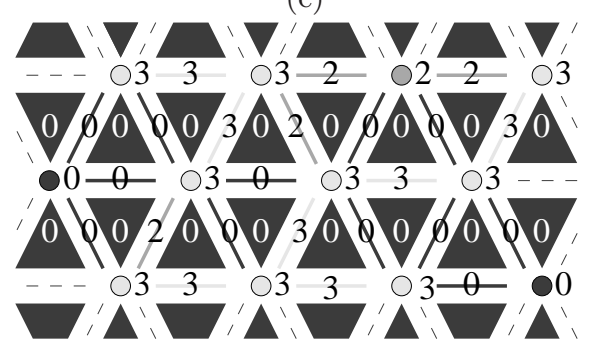

(e)

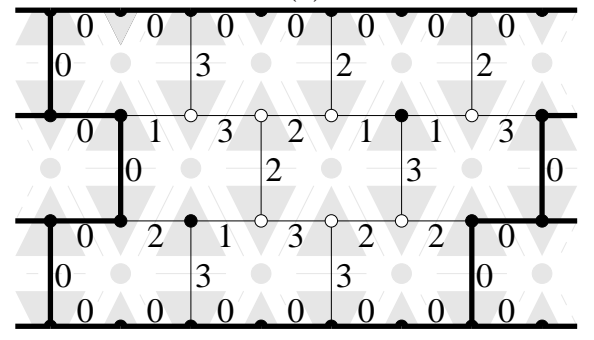

(g)

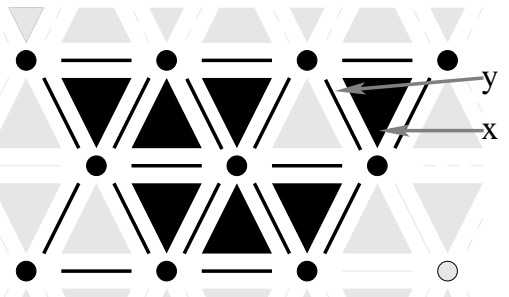

(b)

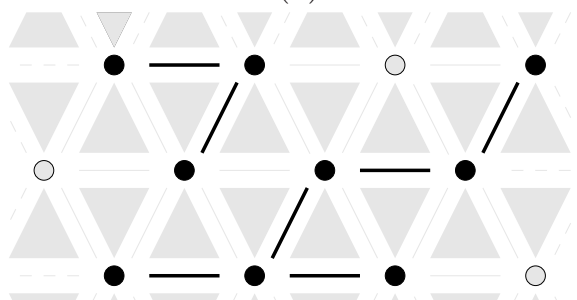

(d)

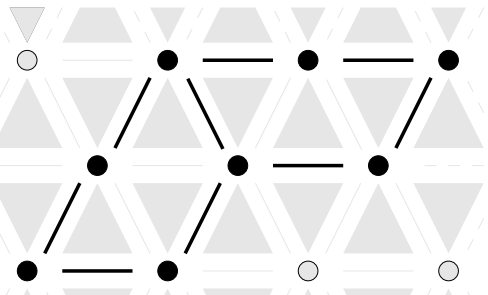

(f)

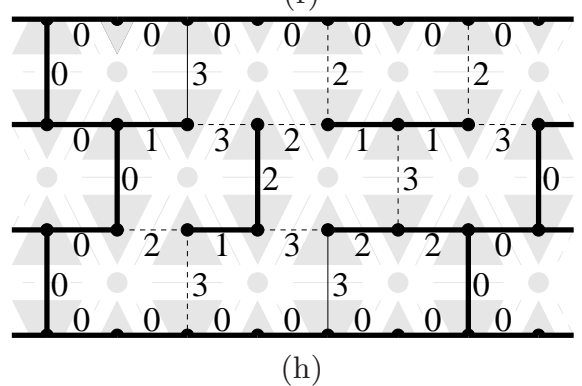

Fig. 5. (a) Representation of a simplicial stack $F$. The gray level of a face corresponds to its altitude which is also indicted by the superimposed number (the faces with no values are supposed to be at altitude 0 ). (b,c,d) The $k$-section of $F$ for respectively $k=1,2,3$. (e) An ultimate 2-collapse $H$ of $F$. (f) A watershed $X$ of $F$ which is equal to the set of all multi-connected faces of $\bar{M}(H)$. (g) The graph $G_{\mathbb{M}}$ and the map $F_{G}$ associated to the manifold $\mathbb{M}$ (light gray) and the stack $F$. In bold, a subgraph $A$ of $G_{\mathbb{M}}$. (h) An MSF relative to $A$ (in bold) and an MSF cut for $A$ (dashed edges). 